\title{
Using complexity science to search for unity in the natural sciences
}

\section{Citation}

Eric Chaisson. 2013. "Using Complexity Science to Search for Unity in the Natural Sciences." In Complexity and the Arrow of Time, ed. Charles H. Lineweaver, Paul C. W. Davies, and Michael Ruse, 68-79. New York: Cambridge University Press.

\section{Published Version}

doi:10.1017/CB09781139225700.006

\section{Permanent link}

http://nrs.harvard.edu/urn-3:HUL.InstRepos:14343113

\section{Terms of Use}

This article was downloaded from Harvard University's DASH repository, and is made available under the terms and conditions applicable to Other Posted Material, as set forth at http:// nrs.harvard.edu/urn-3:HUL.InstRepos:dash.current.terms-of-use\#LAA

\section{Share Your Story}

The Harvard community has made this article openly available.

Please share how this access benefits you. Submit a story.

\section{Accessibility}




\section{Using complexity science to search for unity in the natural sciences}

\section{Eric J. Chaisson}

Nature writ large is a mess. Yet, underlying unities pervade the long and storied, albeit meandering, path from the early universe to civilization on Earth. Evolution is one of those unifiers, incorporating physical, biological, and cultural changes within a broad and inclusive cosmic-evolutionary scenario. Complexity is another such unifier, delineating the growth of structure, function, and diversity within and among galaxies, stars, planets, life, and society throughout natural history. This chapter summarizes a research agenda now underway not only to search for unity in Nature but also, potentially and more fundamentally, to quantify both unceasing evolution and increasing complexity by modeling energy, whose flows through non-equilibrium systems arguably grant opportunities for evolution to create even more complexity.

\section{I COSMIC EVOLUTION}

Truth be told, I am a phenomenologist - neither a theorist studying Nature from first principles (I'm not smart enough) nor an experimentalist actually measuring things (although I used to). My current philosophy of approach aims to observe and characterize Nature thermodynamically, seeking to explicate a scientific worldview that chronicles systematically and sequentially the many varied changes that have occurred from the big bang to humankind on Earth. I call that epic worldview cosmic evolution.

Complexity and the Arrow of Time, ed. Charles H. Lineweaver, Paul C. W. Davies and Michacl Ruse. Published by Cambridge University Press.

(1) Cambridge University Press 2013.
A suggested definition: Cosmic evolution is a grand synthesis of all developmental and generational changes in the assembly and composition of radiation, matter, and life throughout the history of the universe.

The scientific interdiscipline of cosmic evolution as a general study of change is not new; its essence harks back at least 25 centuries to when the philosopher Heraclitus arguably made the best observation ever while noting that "everything flows ... nothing stays." This remarkably simple idea is now confirmed by modern scientific reasoning and much supporting data. I have recently reviewed the status of attempts to undergird the eclectic, integrated scenario of cosmic evolution with quantitative analyses, thereby advancing the topic from subjective colloquy to objective empiricism (Chaisson, 2009a, 2009b).

Academic colleagues often quip that history is "just one damn thing after another", implying that natural history, which goes all the way back in time, comprises myriad and diverse, yet unrelated events. By contrast, I have always regarded natural history expansively and seamlessly as a long and continuous narrative not only incorporating the origin and evolution of a wide spectrum of ordered structures, but also connecting many of them within an overarching framework of understanding. In short, my scientific scholarship firmly roots my work in empirical research, mines data from a wealth of observations across all of space and time, and portrays natural history as an intellectually powerful story that unifies much of what is known about Nature.

Although guiding changes within and among complex systems, evolution itself need not be a complex process. Nor does evolution, as an erratic, rambling activity that is unceasing, uncaring, and unpredictable likely pertain only to life forms. Cosmic evolution extends the central idea of evolution - ascent with modification, generally considered - to embrace all structured systems. And by merging physical, biological, and cultural evolution into a single, intensive paradigm based on everlasting change, cosmic evolution evokes a Platonic ideal 
that the changing, shifting world of natural phenomena and realistic objects masks a deeper, underlying reality of unchanging forms and processes, and that it is these alone that grant true knowledge.

\subsection{ENERGY RATE DENSITY}

All complex systems - whether living or not - are open, organized, non-equilibrated structures that acquire, store, and express energy. This chapter's single goal reiterates and amplifies a previously proposed hypothesis (Chaisson, 2001) that specific energy flow reifies a complexity metric and potential evolutionary driver for all constructive events from the origin of the universe to humans on Earth, as well as for future evolutionary events yet to occur. Energy does seem to be a common currency among such ordered structures; energy flow may well be the most unifying process in science, helping to provide a cogent explanation for the onset, existence, and complexification of a whole array of systems - notably, how they emerge, mature, and terminate during individual lifetimes as well as across multiple generations.

Energy is not likely the only useful metric to measure complexity in complex, evolving systems. Nor do I mean to be critical of alternative schemes, such as information content or entropy production; the literature is replete with controversial claims for such measures, many of them asserted with dogmatic confidence. I have earlier published brief critiques that these and related alternatives are unhelpful for general complexity metrics, their use often narrow, abstract, qualitative, and equivocal (Chaisson, 2001). By contrast, I have embraced the practical concept of energy largely because I can define it, measure it, and clearly express its units. I have furthermore endeavored to quantify this decidedly thermodynamic term in a reliable and consistent manner for a full spectrum of organized systems from spiral galaxies and fusing stars to buzzing bees and redwood trees, indeed to sentient humans and our technological society.

The chosen metric, however, can be neither energy alone, nor even merely energy flow. Life on Earth is surely more complex than any star or galaxy, yet the latter engage vastly more energy than anything now alive on our planet. Accordingly, I have sought to normalize energy flows in complex systems by their inherent mass, thereby enabling more uniform analysis while allowing effective comparison between and among virtually every kind of system encountered in Nature. This, then, has been and continues to be my working hypothesis: mass-normalized energy flow, termed energy rate density and denoted by $\Phi_{m}$, is potentially the most universal process capable of building structures, evolving systems, and creating complexity throughout the universe.

A suggested definition: Energy rate density (also termed power density) is the amount of energy flowing through a system per unit time and per unit mass.

For consistency in this research program's calculations, I have used total energy flowing through the bulk of open systems since all incoming energy passing through such systems is eventually dissipated regardless of the efficiency with which systems utilize energy A more refined analysis might benefit from using either the physicist's "free energy" or the chemist's "enthalpy", although for wellorganized systems internal energy and free energy are nearly the same, and in any case the general results of this study would not likely change much given the ten-order-of-magnitude trend in energy rate density from galaxies to society. Several other recent works have also employed the concept of energy rate density, albeit in more limited venues (e.g., Spier, 2011; Neubauer, 2011).

Figure 4.1 summarizes much recent research on this subject, depicting how physical, biological, and cultural evolution over $\sim 14$ Gy has transformed homogeneous, primordial matter into increasingly intricate systems (Chaisson, 2011a, 2011b). The many graphs show the rise in values of $\Phi_{\mathrm{m}}$ computed for selected systems extant in Nature and of known scientific age. (For specific power units of $\mathrm{W} / \mathrm{kg}$, divide by $10^{4}$.) Values given are typical for the general category to which each system belongs, yet as in any simple, unifying explication of an imperfect universe - especially one like cosmic 

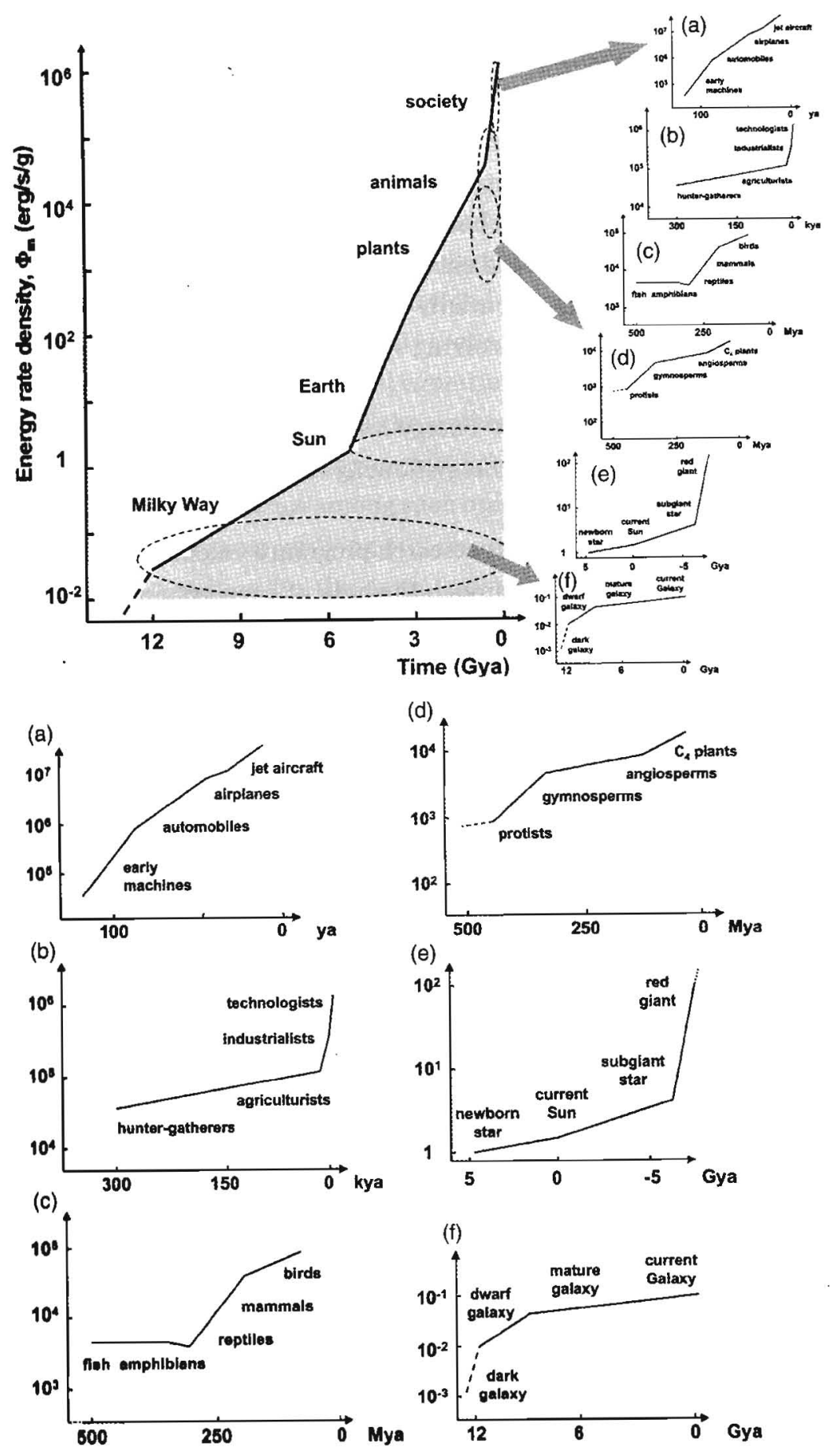

evolution that aspires to address all of Nature - there are moderate variations. And it is likely that from those variations arose the great diversity among complex, evolving systems everywhere.

Better metrics than energy rate density may well describe each of the individual systems within the realms of physical, biological, and cultural evolution that combine to create the greater whole of cosmic evolution, but no other single metric seems capable of uniformly describing them all. The significance of plotting "on the same page" a single quantity for such a wide range of systems observed in Nature should not be overlooked. I am unaware of any other sole quantity that can characterize so extensively a principal system dynamic over $>20$ orders of magnitude in spatial dimension and nearly as many in time.

What seems inherently attractive is that energy flow as a universal process helps suppress entropy within increasingly ordered, localized systems evolving amidst increasingly disordered, surrounding environments, indeed a process that arguably governed the emergence and maturity of our Galaxy, our star, our planet, and ourselves. All accords with the second law of thermodynamics; no violations or circumventions of Nature's most cherished law are evident. If

FIGURE 4.I These many graphs show changing values of energy rate density, $\Phi_{\mathrm{m}}$, for myriad systems observed throughout Nature. The main graph at the top left traces $\Phi_{m}$ for a variety of open, organized, nonequilibrium systems extending from the big bang to humankind. Plotted semi-logarithmically at the time of each system's origin, $\Phi_{m}$ displays a clear increase during the $\sim 14 \mathrm{~Gy}$ history of the universe. The shaded area includes a huge ensemble of changing $\Phi_{\mathrm{m}}$ values as individual systems evolved and complexified. The dashed ovals outline the range in $\Phi_{\mathrm{m}}$ and time bracketing each of the physical, biological, and cultural systems graphed at top right (each magnified at bottom). Rationale for the main plot on the left can be found in Chaisson (2001); data for all the plots on the top right are from Chaisson (2011a, 2011b). Exceptions, outliers, "black swans", or whatever one wants to call those data points that inevitably deviate from the norm, are occasionally evident. The $\Phi_{\mathrm{m}}$ values and historical dates plotted here are estimates, each with ranges and uncertainties; yet it is not their absolute magnitudes and specific quantities that matter as much as their overall trend with the march of time. 
correct, energy itself is a central mechanism of change - a central feature of evolution. And energy rate density is an unambiguous, weighted measure of energy flow enabling us to gauge all complex systems in like manner, as well as to examine how over the course of time some systems were able to command energy and survive, while others apparently could not and did not.

\section{$4 \cdot 3$ COMPLEXITY QUANTIFIED}

Cosmic evolution is not a theory of everything, nor even necessarily a universal theory of evolution; it is, rather, a collection of evolutionary phases - from rudimentary alteration of physical systems, to Darwinian modification of life forms, to Lamarckian reshaping of cultured society - all consistently and fundamentally characterized, at least in part, by mass-normalized energy flow. All complex systems, samples of which are diagnosed below (see, Chaisson, 2011a, $2011 \mathrm{~b}$ ), interact with their environments as matter and energy flow in while wastes flow out, adapt to changing circumstances, and resemble metabolisms at work on many scales. These findings strengthen the time-honored idea that elegantly simple processes underlie the tangled complexity of our richly endowed universe.

A suggested definition: Complexity is a state of intricacy, complication, variety or involvement, as in the interconnected parts of a system - a quality of having many different, interacting components.

\section{Physical evolution}

Stars and galaxies among physical systems generally have energy rate densities that are among the lowest of known organized systems. The latter, including those of dwarf, normal, and active galaxies, display $\Phi_{\mathrm{m}}=0.01-50 \mathrm{erg} / \mathrm{s} / \mathrm{g}$, each type showing clear temporal trends in rising values of $\Phi_{\mathrm{m}}$ while clustering hierarchically, as herewith computed for our Milky Way Galaxy:

- from protogalactic blobs $>12 \mathrm{Gya}\left(\Phi_{\mathrm{m}} \approx 10^{-3} \mathrm{erg} / \mathrm{s} / \mathrm{g}\right)$,

- to widespread dwarf galaxies $\left(\sim 10^{-2}\right)$,

- to mature, normal status $\sim 10$ Gya $(\sim 0.05)$,

- to our Galaxy's current state $(\sim 0.1)$.
Although of lesser complexity and longer duration, the Milky Way is nearly as adaptive and metabolic as any life form - transacting energy while forming new stars, cannibalizing dwarf galaxies, and dissolving older components. Stars, too, adjust their states while evolving during one or more generations, their $\Phi_{\mathrm{m}}$ values rising while they complexify with time. Stellar interiors undergo cycles of nuclear fusion that foster greater thermal and chemical gradients, resulting in increasingly differentiated layers of heavy elements within highly evolved stars. Stellar size, color, brightness, and composition all change while slowly altering the structure of every star, including the Sun, which will eventually be selected out of the population of neighboring stars:

- from early protostar $\sim 5 \mathrm{Gya}\left(\Phi_{\mathrm{m}} \approx 1 \mathrm{erg} / \mathrm{s} / \mathrm{g}\right)$,

- to the main-sequence Sun currently $(\sim 2)$,

- to subgiant status $\sim 6$ Gy in the future $(\sim 4)$,

- to aged red giant near termination $\left(\sim 10^{2}\right)$.

At least as regards energy flow, material resources, and structural integrity while experiencing change, adaptation, and selection, stars have much in common with life. This is not to say that stars are alive, nor that stars evolve in the strict and limited biological sense; most researchers would agree that stars and galaxies develop - as evidenced by systematically rising $\Phi_{\mathrm{m}}$ values.

\section{Biological evolution}

In turn, plants and animals among biological systems regularly exhibit intermediate values of $\Phi_{\mathrm{m}}=10^{3}-10^{5} \mathrm{erg} / \mathrm{s} / \mathrm{g}$. Life does seem to operate optimally within certain limits of temperature, pressure, salinity, etc., and not surprisingly also has an optimal range of normalized energy flow. For plant life on Earth, energy rate densities are much higher than those for galaxies, stars, and planets, as perhaps best illustrated by the evolution of the most dominant process in Earth's biosphere - photosynthesis:

- from microscopic protists $>470 \mathrm{Mya}\left(\Phi_{\mathrm{m}} \approx 10^{3} \mathrm{erg} / \mathrm{s} / \mathrm{g}\right)$,

- to gymnosperms $\sim 350 \mathrm{Mya}\left(\sim 5 \times 10^{3}\right)$,

- to angiosperms $\sim 125 \mathrm{Mya}\left(\sim 7 \times 10^{3}\right)$,

- to highly efficient $\mathrm{C}_{4}$ plants $\sim 30 \mathrm{Mya}\left(\sim 10^{4}\right)$. 
(Onward across the bush of life (or the arrow of time) - cells, tissues, organs, organisms - much the same metric holds for animals while cvolving and complexifying. For adult bodies (much as for brains, which have an order of magnitude larger $\Phi_{m} l$, the temporal trend of rising $\Phi_{\mathrm{m}}$ continues:

- from fish and amphibians $370-500 \mathrm{Mya}\left(\Phi_{\mathrm{m}} \approx 4 \times 10^{3}\right)$,

- to cold-blooded reptiles $\sim 320 \mathrm{Mya}\left(\sim 3 \times 10^{3}\right)$,

- to warm-blooded mammals $\sim 200$ Mya $\left(\sim 4 \times 10^{4}\right)$,

- to birds in flight $\sim 125 \mathrm{Mya}\left(\sim 9 \times 10^{4}\right)$.

Here, system functionality and genetic inheritance, two factors above and beyond mere system structure, help to enhance complexity among animate systems that are clearly living compared to inanimate systems that are clearly not. In either case, energy is fuel for change, apparently (and partly) selecting systems able to utilize increased power densities, while driving others to destruction and extinction - all in accord with neo-Darwinism's widely accepted modern synthesis.

A suggested definition: Life is an open, coherent, spacetime structure kept far from thermodynamic equilibrium by a flow of energy through it - a carbon-based system operating in a water-based medium, with higher forms metabolizing oxygen.

\section{Cultural evolution}

Among cultural systems, advances in technology compare to those of society itself, each of them energy-rich and with $\Phi_{\mathrm{m}} \geq 10^{5} \mathrm{erg} / \mathrm{s} / \mathrm{g}-$ hence plausibly the most complex systems known. Social progress can be tracked, again in terms of energy consumption, for a variety of human-related cultural advances among our human ancestors:

- from hunter-gatherers $\sim 300 \mathrm{kya}\left(\Phi_{\mathrm{m}} \approx 4 \times 10^{4} \mathrm{erg} / \mathrm{s} / \mathrm{g}\right)$,

- to agriculturists $\sim 10 \mathrm{kya}\left(\sim 10^{5}\right)$,

- to industrialists $\sim 200$ ya $\left(\sim 5 \times 10^{5}\right)$,

- to technologists of today $\left(\sim 2 \times 10^{6}\right)$.
Machines, too, and not just computers, but also ordinary motors and engines that typified the fast-paced economy of the twentieth century, can be cast in evolutionary terms - though here the mechanism is less Darwinian than Lamarckian, with the latter's emphasis on accumulation of acquired traits. Either way, energy remains a driver, and with rapidly accelerating pace:

- from primitive machines $\sim 150$ ya $\left(\Phi_{\mathrm{m}} \approx 10^{5} \mathrm{erg} / \mathrm{s} / \mathrm{g}\right)$,

- to the invention of automobiles of $\sim 100$ ya $\left(\sim 10^{6}\right)$,

- to the development of airplanes $\sim 50$ ya $\left(\sim 10^{7}\right)$,

- to computerized jet aircraft of today $\left(\sim 5 \times 10^{7}\right)$.

The road to our present technological society was doubtlessly built with increased energy density used, or per capita energy expended. Increasingly sophisticated technical gadgets, under the Lamarckian pressure of dealer competition and customer selection, do in fact show increases in $\Phi_{m}$ values with product improvement over the years. The cultural evolution of many silicon-based devices now central to our global economy can likewise be traced and their rising $\Phi_{\mathrm{m}}$ values computed, the two - evolution and complexity - paralleling each other once again.

\section{4 SUMMARY}

Complexity science is less empirical and encompassing than many practitioners admit. Traditionally, this subject probes diverse collections of distinct topics, such as cells, ants, economies, and networks, while often appealing to information theory to decipher general principles of mostly biological and social systems that display emergent and adaptive qualities. Such efforts have garnered limited success and an unusual amount of controversy for such a promising new field. Although yielding insightful properties of systems unlikely to be understood by reductionism alone, the real promise of complexity science remains as elusive as when it first arose a generation ago.

This chapter proffers a different strategy. It goes beyond mere words, indeed beyond specialized disciplines, to explore widely, 
deeply, and phenomenologically a process that might characterize complexity quiantitatively across many scientific domains. I have assessed a great array of systems, sought commonalities among them all, and examined a single, uniform metric that arguably quantifies the observed rise of complexity among Nature's many varied systems. The result is an expansive evolutionary scenario not only spanning the known history of time to date but also revealing strong similarities among systems as disparate as stars, life, and society.

Cosmic evolution is more than a subjective, qualitative narration of one unrelated event after another. This inclusive scientific worldview constitutes an objective, quantitative approach toward deciphering much of what comprises organized, material Nature. It addresses the coupled topics of system change and complexity - the temporal advance of the former having contributed to spatial growth of the latter, yet the latter feeding back to make the former increasingly productive. It demonstrates that the basic differences, both within and among many varied complex systems, are of degree, not of kind. And it suggests that optimal ranges of energy rate density grant opportunities for the evolution of complexity; those systems able to adjust, adapt, or otherwise take advantage of such energy flows survive and prosper, while other systems adversely affected by too much or too little energy are non-randomly eliminated. All things considered, I conclude the following:

- Evolution is a universal phenomenon; including changes in physical, biological, and cultural systems, evolution is a unifying principle throughout natural science.

- Energy is a common currency; energy rate density $\left(\Phi_{\mathrm{m}}\right)$ generally correlates with system complexity and may drive, at least in part, the process of evolution itself.

- Selection and adaptation are ubiquitous in Nature; the emergence, maintenance, and fate of all complex systems are often determined, again partly, by their ability to utilize energy.

Physicists tend to notice large trends and general patterns in Nature, often seeking grand unifications or at least global explanations based on few and simple tenets. Biologists, by contrast, concentrate on minute details and intricate mechanisms, often noting quite rightly rare abnormalities in the sweeping generalities. Such dual attitudes perhaps signal the true value of this coarse-grained, phenomenological approach, for only when the devilish details are reconciled with the bigger picture will we be able to call it a "complexity science" that synthesizes both for coherent understanding of ourselves, our world, and our universe.

\section{REFERENCES}

Chaisson, E. J. (2001). Cosmic Evolution: the Rise of Complexity in Nature. Cambridge \& London: Harvard University Press.

Chaisson, E. J. (2009a). Cosmic evolution - state of the science. In S. Dick and M. Lupisella (eds.). Cosmos $\Theta$ Culture. Washington: NASA Press.

Chaisson, E. J. (2009b). Exobiology and complexity. In R. Meyers (ed.). Encyclopedic of Complexity and Systems Science. Berlin: Springer.

Chaisson, E. J. (2011a). Energy rate density as a complexity metric and evolutionary driver. Complexity, 16, 27-40; DOI: 10.1002/cplx.20323.

Chaisson, E. J. (2011b). Energy rate density II: probing further a new complexity metric. Complexity, 17, 44-63; DOI: 10.1002/cplx.20373.

Neubauer, R. L. (2011). Evolution and the Emergent Self: the Rise of Complexity and Behavioral Versatility in Nature. New York: Columbia University Press. Spier, F. (2011). Big History and the Future of Humanity. London: Wiley-Blackwell. 